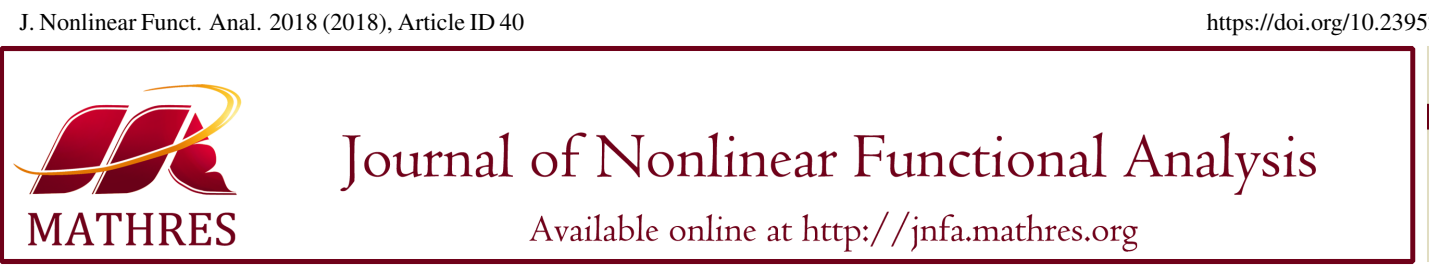

https://doi.org/10.23952/jnfa.2018.40

\title{
VISCOSITY METHODS FOR NONEXPANSIVE AND MONOTONE MAPPINGS IN HILBERT SPACES
}

\author{
YAN $\mathrm{HAO}^{1,2}$ \\ ${ }^{1}$ School of Mathematics, Physics and Information Science, Zhejiang Ocean University, Zhoushan 316022, China \\ ${ }^{2}$ Key Laboratory of Oceanographic Big Data Mining and Application of Zhejiang Province, Zhoushan 316022, China
}

\begin{abstract}
The purpose of this paper is to study variational inclusions, equilibrium problems and fixed point problems via a viscosity iterative algorithm. Strong convergence of the viscosity iterative algorithm is obtained in a Hilbert space.
\end{abstract}

Keywords. Inclusion problem; Nonexpansive mapping; Equilibrium problem, Iteration method; Variational inequality.

2010 Mathematics Subject Classification. 47H05, 47J25, 90C25.

\section{INTRODUCTION-PRELIMINARIES}

The study on convex feasibility problems is an important branch of nonlinear analysis optimization theory. Numerous problems in physics, transportation, signal process and economics are reduced to find a solution to a convex feasibility problem, which cover inclusion problems, equilibrium problems, fixed point problems, variational inequalities and so on. The motivation for this subject is mainly due to its possible applications to mathematical modeling of concrete complex problems; see [1]-[5] and the references therein. Solving such problems, we have to obtain some solution which is simultaneously a solution of two or more subproblems or a solution of one subproblem on solution sets of another subproblem; see [6]-[14] and the references therein.

Let $H$ be a real Hilbert space with inner product $\langle\cdot, \cdot\rangle$ and norm $\|\cdot\|$. Let $C$ be a nonempty, closed, and convex subset of $H$ and let $\operatorname{Proj}_{C}$ be the metric projection from $H$ onto $C$.

Let $A: C \rightarrow H$ be a mapping. Recall that $A$ is said to be monotone iff

$$
\langle A x-A y, x-y\rangle \geq 0, \quad \forall x, y \in C .
$$

Recall that $A$ is said to be inverse-strongly monotone iff there exists a constant $\alpha>0$ such that

$$
\langle A x-A y, x-y\rangle \geq \alpha\|A x-A y\|^{2}, \quad \forall x, y \in C .
$$

For such a case, $A$ is also said to be $\alpha$-inverse-strongly monotone.

E-mail address: zjhaoyan@aliyun.com.

Received August 23, 2018; Accepted December 14, 2018.

(C) 2018 Journal of Nonlinear Functional Analysis 
Recall that the celebrated classical variational inequality is to find an $x \in C$ such that

$$
\langle y-x, A x\rangle \geq 0, \quad \forall y \in C
$$

In this paper, we use $\operatorname{VI}(C, A)$ to denote the solution set of (1.1). It is known that $x \in C$ is a solution of variational inequality (1.1) iff $x$ is a solution of the fixed point equation $\operatorname{Proj}_{C}(I-r A) x=x$, where $r>0$ is a constant and $I$ is the identity mapping on $H$. Recently, fixed point algorithms have been employed to study solutions of of variational inequality $(1.1)$; see $[6,11,15,16]$ and the references therein.

Let $F$ be a bifunction of $C \times C$ into $\mathbb{R}$, where $\mathbb{R}$ denotes the set of real numbers. We consider the following generalized equilibrium problem.

$$
\text { Find } x \in C \text { such that } F(x, y) \geq 0, \quad \forall y \in C \text {. }
$$

In this paper, we use $E P(F)$ to denote the solution set of equilibrium problem (1.2). The equilibrium problem provides us with a natural, unified, and general framework to study a wide class of problems arising in finance, economics, network analysis, transportation, elasticity and optimization. This theory has witnessed an explosive growth in theoretical advances and applications. Recently, numerical methods have been introduced and studied for solutions of equilibrium problem $(1.2)$; see $[3,6,9,10,17,18]$ and the references therein.

To study the equilibrium problem, we may assume that $F$ satisfies the following conditions:

(A1) $F(x, y)+F(y, x) \leq 0$ for all $x, y \in C$;

(A2) $F(x, x)=0$ for all $x \in C$;

(A3) for each $x \in C, y \mapsto F(x, y)$ is convex and weakly lower semi-continuous;

(A4) for each $x, y, z \in C$,

$$
\limsup _{t \downarrow 0} F(t z+(1-t) x, y) \leq F(x, y) .
$$

Let $T: C \rightarrow C$ be a mapping. In this paper, we use $F i x(T)$ to denote the fixed point set of $T$. Recall that $T$ is said to be contractive iff there exists a constant $\alpha \in(0,1)$ such that

$$
\|T x-T y\| \leq \alpha\|x-y\|, \quad \forall x, y \in C .
$$

For such a case, $T$ is also said to be $\alpha$-contractive. Recall that $T$ is said to be nonexpansive iff

$$
\|T x-T y\| \leq\|x-y\|, \quad \forall x, y \in C .
$$

It is known that the fixed point set of nonexpansive mappings is nonempty provided that the subset $C$ is bounded, convex and closed. Finding an optimal point in common fixed/zero point sets of a family of nonlinear mappings is very important, which occurs frequently in various areas of mathematical sciences and engineering; see [19]-[24] and the references therein. 
Let $\left\{S_{i}: C \rightarrow C\right\}$ be a family of infinitely nonexpansive mappings and let $\left\{\gamma_{i}\right\}$ be a nonnegative real sequence with $0 \leq \gamma_{i}<1, \forall i \geq 1$. For $n \geq 1$, define a mapping $W_{n}: C \rightarrow C$ as follows:

$$
\begin{aligned}
& V_{n, n+1}=I, \\
& V_{n, n}=\left(1-\gamma_{n}\right) I+\gamma_{n} S_{n} V_{n, n+1}, \\
& V_{n, n-1}=\left(1-\gamma_{n-1}\right) I+\gamma_{n-1} S_{n-1} V_{n, n}, \\
& \vdots \\
& V_{n, k}=\left(1-\gamma_{k}\right) I+\gamma_{k} S_{k} V_{n, k+1}, \\
& V_{n, k-1}=\left(1-\gamma_{k-1}\right) I+\gamma_{k-1} S_{k-1} V_{n, k}, \\
& \vdots \\
& V_{n, 2}=\left(1-\gamma_{2}\right) I+\gamma_{2} S_{2} V_{n, 3}, \\
& W_{n}=V_{n, 1}=\left(1-\gamma_{1}\right) I+\gamma_{1} S_{1} V_{n, 2} .
\end{aligned}
$$

Such a mapping $W_{n}$ is nonexpansive from $C$ to $C$ and it is called a $W$-mapping generated by $S_{n}, S_{n-1}, \ldots, S_{1}$ and $\gamma_{n}, \gamma_{n-1}, \ldots, \gamma_{1}$, see [20] and the references therein.

Recall that a set-valued mapping $M: H \rightrightarrows H$ is said to be monotone iff, for all $x, y \in H, f \in M x$ and $g \in M y$ imply $\langle x-y, f-g\rangle>0 . M$ is a maximal mapping iff the graph $\operatorname{Graph}(M)$ of $M$ is not properly contained in the graph of any other monotone mapping. It is known that a monotone mapping $M$ is maximal if and only if, for any $(x, f) \in H \times H,\langle x-y, f-g\rangle \geq 0$, for all $(y, g) \in \operatorname{Graph}(M)$ implies $f \in R x$.

For a maximal monotone operator $M$ on $H$, and $r>0$, we may define the single-valued resolvent $J_{r}$ : $H \rightarrow \operatorname{Dom}(M)$, where $\operatorname{Dom}(M)$ denotes the domain of $M$. It is known that $J_{r}$ is firmly nonexpansive, that is, $\left\|J_{r} x-J_{r} y\right\|^{2} \leq\left\langle J_{r} x-J_{r} y, x-y\right\rangle, \forall x, y \in H$, and $\operatorname{Fix}\left(J_{r}\right)=M^{-1}(0)$, where $\operatorname{Fix}\left(J_{r}\right):=\{x \in \operatorname{Dom}(M)$ : $\left.x=J_{r} x\right\}$, and $M^{-1}(0):=\{x \in H: 0 \in M x\}$.

Splitting methods have recently received much attention due to the fact that many nonlinear problems arising in applied areas such as signal processing, image recovery, and machine learning are mathematically modeled as a nonlinear operator equation and this operator is decomposed as the sum of two nonlinear operators. Splitting methods for linear equations were introduced by Peaceman and Rachford [1] and Douglas and Rachford [2]. Extensions to nonlinear equations in Hilbert spaces were carried out by Kellogg [25] and Lions and Mercier [26]. The central problem is to iteratively find a zero of the sum of two monotone operators $A$ and $M$ in a Hilbert space $H$, namely, a solution to the inclusion problem $0 \in(A+M) x$. Many problems can be formulated as a problem of the inclusion problem, such as, stationary solutions to initial value problems of evolution equations. In this paper, we investigate an equilibrium problem, a inclusion problem and a common fixed point problem via a viscosity approximation method. Strong convergence theorems of common solutions to the problems are established in the framework of Hilbert spaces.

Lemma 1.1 ([20]). Let $H$ be a Hilbert space and let $C$ be a nonempty convex closed subset of $H$. Let $\left\{S_{i}: C \rightarrow C\right\}$ be a family of infinitely nonexpansive mappings with a nonempty common fixed point set and let $\left\{\gamma_{i}\right\}$ be a real sequence such that $0<\gamma_{i} \leq l<1$, where $l$ is some real number, $\forall i \geq 1$. Then

(1) $W_{n}$ is nonexpansive and Fix $\left(W_{n}\right)=\cap_{i=1}^{\infty} F i x\left(S_{i}\right)$, for each $n \geq 1$; 
(2) for each $x \in C$ and for each positive integer $k$, the limit $\lim _{n \rightarrow \infty} V_{n, k}$ exists.

(3) $W: C \rightarrow C$ defined by

$$
W x:=\lim _{n \rightarrow \infty} W_{n} x=\lim _{n \rightarrow \infty} V_{n, 1} x, \quad x \in C
$$

is a nonexpansive mapping satisfying $\operatorname{Fix}(W)=\cap_{i=1}^{\infty} F i x\left(S_{i}\right)$ and it is called the $W$-mapping generated by $S_{1}, S_{2}, \ldots$ and $\gamma_{1}, \gamma_{2}, \ldots$

Lemma 1.2 ([11]). Let $H$ be a Hilbert space and let $C$ be a nonempty convex closed subset of $H$. Let $\left\{S_{i}: C \rightarrow C\right\}$ be a family of infinitely nonexpansive mappings with a nonempty common fixed point set and let $\left\{\gamma_{i}\right\}$ be a real sequence such that $0<\gamma_{i} \leq l<1, \forall i \geq 1$. If $K$ is any bounded subset of $C$, then $\lim _{n \rightarrow \infty} \sup _{x \in K}\left\|W x-W_{n} x\right\|=0$.

From now on, we always assume that $0<\gamma_{i} \leq l<1, \forall i \geq 1$.

Lemma 1.3 ([27]). Let $H$ be a Hilbert space and let $C$ be a nonempty convex closed subset of $H$. Let $F: C \times C \rightarrow \mathbb{R}$ be a bifunction satisfying (A1)-(A4). Then, for any $s>0$ and $x \in H$, there exists $z \in C$ such that

$$
F(z, y)+\frac{1}{s}\langle y-z, z-x\rangle \geq 0, \quad \forall y \in C .
$$

Define a mapping $\operatorname{Res}_{s}: H \rightarrow C$ as follows:

$$
\operatorname{Res}_{s} x=\left\{z \in C: F(z, y)+\frac{1}{s}\langle y-z, z-x\rangle \geq 0, \quad \forall y \in C\right\}, \quad x \in H .
$$

Then

(a) Res is single-valued firmly nonexpansive, i.e.,

$$
\left\|\operatorname{Res}_{s} x-\operatorname{Res}_{s} y\right\|^{2} \leq\left\langle\operatorname{Res}_{s} x-\operatorname{Res}_{s} y, x-y\right\rangle, \quad \forall x, y \in H,
$$

(b) $\operatorname{EP}(F)=F i x\left(\operatorname{Res}_{s}\right)$ is closed and convex.

Lemma 1.4 ([28]). Let $H$ be a Hilbert space and and let $M$ be a maximal monotone operator. For $r>0$, $s>0$ and $x \in H$, we have

$$
J_{r} y=J_{s}\left(\frac{s}{r} y+\frac{r-s}{r} J_{r} y\right)
$$

where $J_{r}=(I+r M)^{-1}$ and $J_{s}=(I+s M)^{-1}$.

Lemma 1.5 ([24]). Let $H$ be a Hilbert space. Let $\left\{x_{n}\right\}$ and $\left\{y_{n}\right\}$ be bounded sequences in $H$. Let $x_{n+1}=$ $\left(1-a_{n}\right) y_{n}+a_{n} x_{n}$, where $\left\{a_{n}\right\}$ is a sequence in $(0,1)$ such that $0<\liminf _{n \rightarrow \infty} a_{n} \leq \limsup _{n \rightarrow \infty} a_{n}<1$. If

$$
\underset{n \rightarrow \infty}{\limsup }\left(\left\|y_{n+1}-y_{n}\right\|-\left\|x_{n+1}-x_{n}\right\|\right) \leq 0
$$

then $\lim _{n \rightarrow \infty}\left\|y_{n}-x_{n}\right\|=0$.

Lemma 1.6 ([29]). Let $H$ be a Hilbert space. Let $A: C \rightarrow H$ be a mapping and let $M: H \rightrightarrows H$ be a maximal monotone operator. Then Fix $\left((I+r M)^{-1}(I-r A)\right)=(M+A)^{-1}(0)$, where $r$ is some positive real number.

Lemma 1.7 ([30]). Let $\left\{\alpha_{n}\right\}$ be a sequence of nonnegative real numbers such that $\alpha_{n+1} \leq\left(1-\gamma_{n}\right) \alpha_{n}+$ $\delta_{n}$, where $\left\{\gamma_{n}\right\}$ is a sequence in $(0,1)$ and $\left\{\delta_{n}\right\}$ is a sequence such that $\sum_{n=1}^{\infty} \gamma_{n}=\infty$ and $\limsup _{n \rightarrow \infty} \delta_{n} / \gamma_{n} \leq$ 0. Then $\lim _{n \rightarrow \infty} \alpha_{n}=0$. 


\section{MAIN RESULTS}

Theorem 2.1. Let $H$ be a real Hilbert space and let $C$ be a nonempty convex closed subset of $H$. Let $f: C \rightarrow C$ be a $\kappa$-contraction. Let $A: C \rightarrow H$ be an $\alpha$-inverse-strongly monotone mapping and let $M: H \rightrightarrows H$ be a maximal monotone operator such that Dom $(M) \subset C$. Let $S_{i}$ be a nonexpansive mapping for each $i \geq 1$ and let $F$ be a bifunction from $C \times C$ to $\mathbb{R}$ which satisfies (A1)-(A4). Let $\left\{x_{n}\right\}$ be a sequence generated in the process:

$$
\left\{\begin{array}{l}
x_{1} \in C, \text { chosen arbitrarily, } \\
u_{n}=J_{r_{n}}\left(y_{n}-r_{n} A y_{n}\right), \\
x_{n+1}=\alpha_{n} f\left(W_{n} x_{n}\right)+\beta_{n} x_{n}+\gamma_{n} W_{n} J_{r_{n}}\left(u_{n}-r_{n} A u_{n}\right), \quad \forall n \geq 1
\end{array}\right.
$$

where $y_{n}$ is in $C$ such that

$$
F\left(y_{n}, y\right)+\frac{1}{s_{n}}\left\langle y-y_{n}, y_{n}-x_{n}\right\rangle \geq 0, \quad \forall y \in C,
$$

$W_{n}$ is defined by (1.3), $\left\{\alpha_{n}\right\},\left\{\beta_{n}\right\}$ and $\left\{\gamma_{n}\right\}$ are sequences in $(0,1)$ such that $\alpha_{n}+\beta_{n}+\gamma_{n}=1$ and $\left\{r_{n}\right\}$ and $\left\{s_{n}\right\}$ are positive number sequences. Assume that the above control sequences satisfy the following restrictions: $\lim _{n \rightarrow \infty}\left|s_{n}-s_{n+1}\right|=\lim _{n \rightarrow \infty}\left|r_{n}-r_{n+1}\right|=0,0<\liminf _{n \rightarrow \infty} \beta_{n} \leq \limsup _{n \rightarrow \infty} \beta_{n}<$ $1, \lim _{n \rightarrow \infty} \alpha_{n}=0$ and $\sum_{n=1}^{\infty} \alpha_{n}=\infty, 0<r \leq r_{n} \leq r^{\prime}<2 \alpha, 0<s \leq s_{n} \leq s^{\prime}<2 \beta$, where $r$, $r^{\prime}$, $s$ and $s^{\prime}$ are four real numbers. If $\cap_{i=1}^{\infty} F\left(S_{i}\right) \cap(A+M)^{-1}(0) \cap E P(F) \neq \emptyset$. Then $\left\{x_{n}\right\}$ converges strongly to $\bar{x}=\operatorname{Proj}_{\cap_{i=1}^{\infty} F\left(S_{i}\right) \cap(A+M)^{-1}(0) \cap E P(F)} f(\bar{x})$.

Proof. Fix $x^{*} \in \cap_{i=1}^{\infty} F\left(S_{i}\right) \cap(A+M)^{-1}(0) \cap E P(F)$. It follows from Lemma 1.3 that $R_{S_{n}} x^{*}=x^{*}$. We also have $x^{*}=J_{r_{n}}\left(I-r_{n} A\right) x^{*}$ and $x^{*}=S_{i} x^{*}$, for each $i \geq 1$. For $\forall x, y \in C$, we have

$$
\begin{aligned}
& \left\|\left(I-r_{n} A\right) x-\left(I-r_{n} A\right) y\right\|^{2} \\
& =\|x-y\|^{2}-2 r_{n}\langle x-y, A x-A y\rangle+r_{n}^{2}\|A x-A y\|^{2} \\
& \leq\|x-y\|^{2}-r_{n}\left(2 \alpha-r_{n}\right)\|A x-A y\|^{2} .
\end{aligned}
$$

Taking into account that $0<r \leq r_{n} \leq r^{\prime}<2 \alpha$, we see that $I-r_{n} A$ is nonexpansive. It follows that

$$
\begin{aligned}
\left\|u_{n}-x^{*}\right\| & \leq\left\|J_{r_{n}}\left(I-r_{n} A\right) R_{S_{n}} x_{n}-x^{*}\right\| \\
& \leq\left\|\left(I-r_{n} A\right) R_{S_{n}} x_{n}-x^{*}\right\| \\
& \leq\left\|R_{S_{n}} x_{n}-x^{*}\right\| \\
& \leq\left\|x_{n}-x^{*}\right\| .
\end{aligned}
$$

So,

$$
\begin{aligned}
\left\|x_{n+1}-x^{*}\right\| & \leq \alpha_{n}\left\|f\left(W_{n} x_{n}\right)-x^{*}\right\|+\beta_{n}\left\|x_{n}-x^{*}\right\|+\gamma_{n}\left\|W_{n} u_{n}-x^{*}\right\| \\
& \leq \alpha_{n} \kappa\left\|W_{n} x_{n}-x^{*}\right\|+\alpha_{n}\left\|f\left(x^{*}\right)-x^{*}\right\|+\left(1-\alpha_{n}\right)\left\|u_{n}-x^{*}\right\| \\
& \leq \alpha_{n} \kappa\left\|W_{n} x_{n}-x^{*}\right\|+\alpha_{n}\left\|f\left(x^{*}\right)-x^{*}\right\|+\left(1-\alpha_{n}\right)\left\|x_{n}-x^{*}\right\| \\
& \leq \alpha_{n}(1-\kappa) \frac{\left\|f\left(x^{*}\right)-x^{*}\right\|}{(1-\kappa)}+\left(1-\alpha_{n}(1-\kappa)\right)\left\|x_{n}-x^{*}\right\|,
\end{aligned}
$$


which yields that $\left\|x_{n}-x^{*}\right\| \leq \max \left\{\left\|x_{1}-x^{*}\right\|, \frac{\left\|f\left(x^{*}\right)-x^{*}\right\|}{1-\kappa}\right\}$. This shows that $\left\{x_{n}\right\}$ is bounded. From Lemma 1.4 , we find that

$$
\begin{aligned}
& \left\|J_{r_{n+1}}\left(y_{n}-r_{n} A y_{n}\right)-J_{r_{n}}\left(y_{n}-r_{n} A y_{n}\right)\right\| \\
& =\left\|J_{r_{n}}\left(\frac{r_{n}}{r_{n+1}}\left(y_{n}-r_{n} A y_{n}\right)+\left(1-\frac{r_{n}}{r_{n+1}}\right) J_{r_{n+1}}\left(y_{n}-r_{n} A y_{n}\right)\right)-J_{r_{n}}\left(y_{n}-r_{n} A y_{n}\right)\right\| \\
& \left.\leq\left|1-\frac{r_{n}}{r_{n+1}}\right| \| J_{r_{n+1}}\left(y_{n}-r_{n} A y_{n}\right)\right)-\left(y_{n}-r_{n} A y_{n}\right) \|
\end{aligned}
$$

Since $J_{r_{n}}$ is firmly nonexpansive, one finds from (2.1) that

$$
\begin{aligned}
& \left\|u_{n+1}-u_{n}\right\| \\
& \leq\left\|J_{r_{n+1}}\left(y_{n+1}-r_{n+1} A y_{n+1}\right)-J_{r_{n+1}}\left(y_{n}-r_{n} A y_{n}\right)\right\| \\
& \quad+\left\|J_{r_{n+1}}\left(y_{n}-r_{n} A y_{n}\right)-J_{r_{n}}\left(y_{n}-r_{n} A y_{n}\right)\right\| \\
& \leq\left\|y_{n+1}-y_{n}\right\|+\left|r_{n}-r_{n+1}\right|\left\|A y_{n}\right\|+\left\|J_{r_{n+1}}\left(y_{n}-r_{n} A y_{n}\right)-J_{r_{n}}\left(y_{n}-r_{n} A y_{n}\right)\right\| \\
& \leq\left\|y_{n+1}-y_{n}\right\|+\left|r_{n}-r_{n+1}\right| \Theta_{1},
\end{aligned}
$$

where $\Theta_{1}$ is an appropriate constant. Putting $\xi_{n}=J_{r_{n}}\left(u_{n}-r_{n} A u_{n}\right)$, we find from (2.2) that

$$
\left\|\xi_{n+1}-\xi_{n}\right\| \leq\left\|y_{n+1}-y_{n}\right\|+\left|r_{n}-r_{n+1}\right| \Theta_{2}
$$

where $\Theta_{2}$ is an appropriate constant. From Lemma 1.3, we find that

$$
s_{n+1} F\left(y_{n+1}, y\right)+\left\langle y-y_{n+1}, y_{n+1}-x_{n+1}\right\rangle \geq 0, \quad \forall y \in C,
$$

and

$$
s_{n} F\left(y_{n}, y\right)+\left\langle y-y_{n}, y_{n}-x_{n}\right\rangle \geq 0, \quad \forall y \in C .
$$

Putting $y=y_{n}$ and $y=y_{n+1}$ into the above two inequalities, we find that

$$
\left\langle y_{n+1}-y_{n}, \frac{y_{n}-x_{n}}{s_{n}}-\frac{y_{n+1}-x_{n+1}}{s_{n+1}}\right\rangle \geq 0 .
$$

This implies that

$$
\begin{aligned}
& \left.\left\|y_{n+1}-y_{n}\right\|\left(\left\|x_{n+1}-x_{n}\right\|+\left|1-\frac{s_{n}}{s_{n+1}}\right| \| y_{n+1}-x_{n+1}\right) \|\right) \\
& \geq\left\langle y_{n+1}-y_{n}, x_{n+1}-x_{n}+\left(1-\frac{s_{n}}{s_{n+1}}\right)\left(y_{n+1}-x_{n+1}\right)\right\rangle \\
& \geq\left\|y_{n+1}-y_{n}\right\|^{2} .
\end{aligned}
$$

It follows that

$$
\left\|x_{n+1}-x_{n}\right\|+\left|s_{n+1}-s_{n}\right| \Theta_{3} \geq\left\|y_{n+1}-y_{n}\right\|,
$$

where $\Theta_{3}$ is an appropriate constant. Combining (2.3) with (2.4), we arrive at

$$
\left\|\xi_{n+1}-\xi_{n}\right\| \leq\left\|x_{n+1}-x_{n}\right\|+\left(\left|s_{n+1}-s_{n}\right|+\left|r_{n}-r_{n+1}\right|\right) \Theta_{4},
$$

where $\Theta_{4}$ is an appropriate constant. Note that

$$
\begin{aligned}
& \left\|W_{n+1} \xi_{n+1}-W_{n} \xi_{n}\right\| \\
& \leq\left\|W_{n+1} \xi_{n+1}-W \xi_{n+1}\right\|+\left\|W \xi_{n}-W_{n} \xi_{n}\right\|+\left\|W \xi_{n+1}-W \xi_{n}\right\| \\
& \leq \sup _{x \in K}\left\{\left\|W_{n+1} x-W x\right\|+\left\|W x-W_{n} x\right\|\right\}+\left\|\xi_{n+1}-\xi_{n}\right\|,
\end{aligned}
$$


where $K$ is the bounded subset of $C$. Letting $x_{n+1}=\left(1-\beta_{n}\right) \pi_{n}+\beta_{n} x_{n}$, we see that

$$
\begin{aligned}
\pi_{n+1}-\pi_{n}= & \frac{\alpha_{n+1} f\left(W_{n+1} x_{n+1}\right)+\left(1-\beta_{n+1}-\alpha_{n+1}\right) W_{n+1} \xi_{n+1}}{1-\beta_{n+1}} \\
& -\frac{\alpha_{n} f\left(W_{n} x_{n}\right)+\left(1-\beta_{n}-\alpha_{n}\right) W_{n} \xi_{n}}{1-\beta_{n}} \\
= & \frac{\alpha_{n+1}\left(f\left(W_{n+1} x_{n+1}\right)-W_{n+1} \xi_{n+1}\right)}{1-\beta_{n+1}}+W_{n+1} \xi_{n+1} \\
& -\frac{\alpha_{n}\left(f\left(W_{n} x_{n}\right)-W_{n} \xi_{n}\right)}{1-\beta_{n}}-W_{n} \xi_{n} .
\end{aligned}
$$

It follows from (2.6) that

$$
\begin{aligned}
&\left\|\pi_{n+1}-\pi_{n}\right\| \\
& \leq \frac{\alpha_{n}}{1-\beta_{n}}\left\|f\left(W_{n} x_{n}\right)-W_{n} \xi_{n}\right\|+\frac{\alpha_{n+1}}{1-\beta_{n+1}}\left\|f\left(W_{n+1} x_{n+1}\right)-W_{n+1} \xi_{n+1}\right\| \\
&+\left\|W_{n+1} \xi_{n+1}-W_{n} \xi_{n}\right\| \\
& \leq \frac{\alpha_{n}}{1-\beta_{n}}\left\|f\left(W_{n} x_{n}\right)-W_{n} \xi_{n}\right\|+\frac{\alpha_{n+1}}{1-\beta_{n+1}}\left\|f\left(W_{n+1} x_{n+1}\right)-W_{n+1} \xi_{n+1}\right\| \\
&+\sup _{x \in K}\left\{\left\|W_{n+1} x-W x\right\|+\left\|W x-W_{n} x\right\|\right\}+\left\|\xi_{n+1}-\xi_{n}\right\| .
\end{aligned}
$$

From (2.5) and (2.7), we arrive at

$$
\begin{aligned}
& \left\|\pi_{n+1}-\pi_{n}\right\|-\left\|x_{n+1}-x_{n}\right\| \\
& \leq \frac{\alpha_{n}}{1-\beta_{n}}\left\|f\left(W_{n} x_{n}\right)-W_{n} \xi_{n}\right\|+\frac{\alpha_{n+1}}{1-\beta_{n+1}}\left\|f\left(W_{n+1} x_{n+1}\right)-W_{n+1} \xi_{n+1}\right\| \\
& \quad+\sup _{x \in K}\left\{\left\|W_{n+1} x-W x\right\|+\left\|W x-W_{n} x\right\|\right\}+\left(\left|s_{n+1}-s_{n}\right|+\left|r_{n}-r_{n+1}\right|\right) \Theta_{4} .
\end{aligned}
$$

This yields that

$$
\limsup _{n \rightarrow \infty}\left(\left\|\pi_{n+1}-\pi_{n}\right\|-\left\|x_{n+1}-x_{n}\right\|\right) \leq 0 .
$$

From Lemma 1.5, we find that $\lim _{n \rightarrow \infty}\left\|x_{n}-\pi_{n}\right\|=0$, which yields from the definition of $\pi_{n}$ that

$$
\lim _{n \rightarrow \infty}\left\|x_{n+1}-x_{n}\right\|=0 \text {. }
$$

Now, we are in a position to show $\limsup _{n \rightarrow \infty}\left\langle f(\bar{x})-\bar{x}, x_{n}-z\right\rangle \leq 0$, where

$$
\bar{x}=\operatorname{Proj}_{\cap_{i=1}^{\infty} F\left(S_{i}\right) \cap(A+M)^{-1}(0) \cap E P(F)} f(\bar{x}) .
$$

To prove this, we choose a subsequence $\left\{x_{n_{i}}\right\}$ of $\left\{x_{n}\right\}$ such that

$$
\limsup _{n \rightarrow \infty}\left\langle f(\bar{x})-\bar{x}, x_{n}-\bar{x}\right\rangle=\lim _{i \rightarrow \infty}\left\langle f(\bar{x})-\bar{x}, x_{n_{i}}-\bar{x}\right\rangle .
$$

Since $\left\{x_{n_{i}}\right\}$ is bounded, without loss of generality, we may assume that $x_{n_{i}} \rightarrow q$. For any $x^{*} \in \cap_{i=1}^{\infty} F\left(S_{i}\right) \cap$ $(A+M)^{-1}(0) \cap E P(F)$, we see that

$$
\begin{aligned}
\left\|y_{n}-x^{*}\right\|^{2} & \leq\left\|\operatorname{Res}_{S_{n}} x_{n}-\operatorname{Res}_{S_{n}} x^{*}\right\|^{2} \\
& \leq\left\langle\operatorname{Res}_{S_{n}} x_{n}-\operatorname{Res}_{S_{n}} x^{*}, x_{n}-x^{*}\right\rangle \\
& \leq \frac{\left\|y_{n}-x^{*}\right\|^{2}+\left\|x_{n}-x^{*}\right\|^{2}-\left\|x_{n}-y_{n}\right\|^{2}}{2},
\end{aligned}
$$


which yields that

$$
\left\|y_{n}-x^{*}\right\|^{2} \leq\left\|x_{n}-x^{*}\right\|^{2}-\left\|x_{n}-y_{n}\right\|^{2} .
$$

On the other hand, one has

$$
\begin{aligned}
\left\|x_{n+1}-x^{*}\right\|^{2} & \leq \alpha_{n}\left\|f\left(W_{n} x_{n}\right)-x^{*}\right\|^{2}+\beta_{n}\left\|x_{n}-x^{*}\right\|^{2}+\gamma_{n}\left\|W_{n} \xi_{n}-x^{*}\right\|^{2} \\
& \leq \alpha_{n}\left\|f\left(W_{n} x_{n}\right)-x^{*}\right\|^{2}+\beta_{n}\left\|x_{n}-x^{*}\right\|^{2}+\gamma_{n}\left\|u_{n}-x^{*}\right\|^{2} \\
& \leq \alpha_{n}\left\|f\left(W_{n} x_{n}\right)-x^{*}\right\|^{2}+\left\|x_{n}-x^{*}\right\|^{2}-\gamma_{n}\left\|x_{n}-y_{n}\right\|^{2} .
\end{aligned}
$$

It follows that

$$
\begin{aligned}
\gamma_{n}\left\|x_{n}-y_{n}\right\|^{2} & \leq \alpha_{n}\left\|f\left(W_{n} x_{n}\right)-x^{*}\right\|^{2}+\left\|x_{n}-x^{*}\right\|^{2}-\left\|x_{n+1}-x^{*}\right\|^{2} \\
& \leq \alpha_{n}\left\|f\left(W_{n} x_{n}\right)-x^{*}\right\|^{2}+\left\|x_{n+1}-x_{n}\right\|\left(\left\|x_{n}-x^{*}\right\|+\left\|x_{n+1}-x^{*}\right\|\right) .
\end{aligned}
$$

Since

$$
0<\liminf _{n \rightarrow \infty} \beta_{n} \leq \limsup _{n \rightarrow \infty} \beta_{n}<1,
$$

and $\lim _{n \rightarrow \infty} \alpha_{n}=0$, we arrive at

$$
\lim _{n \rightarrow \infty}\left\|y_{n}-x_{n}\right\|=0
$$

Note that

$$
\begin{aligned}
\left\|u_{n}-x^{*}\right\|^{2} & \leq\left\|\left(I-r_{n} A\right) y_{n}-\left(I-r_{n} A\right) x^{*}\right\|^{2} \\
& =\left\|y_{n}-x^{*}\right\|^{2}-2 r_{n}\left\langle y_{n}-x^{*}, A y_{n}-A x^{*}\right\rangle+r_{n}^{2}\left\|A y_{n}-A x^{*}\right\|^{2} \\
& \leq\left\|x_{n}-x^{*}\right\|^{2}-r_{n}\left(2 \alpha-r_{n}\right)\left\|A y_{n}-A x^{*}\right\|^{2},
\end{aligned}
$$

which implies that

$$
\begin{aligned}
\left\|x_{n+1}-x^{*}\right\|^{2} & \leq \alpha_{n}\left\|f\left(W_{n} x_{n}\right)-x^{*}\right\|^{2}+\beta_{n}\left\|x_{n}-x^{*}\right\|^{2}+\gamma_{n}\left\|W_{n} \xi_{n}-x^{*}\right\|^{2} \\
& \leq \alpha_{n}\left\|f\left(W_{n} x_{n}\right)-x^{*}\right\|^{2}+\beta_{n}\left\|x_{n}-x^{*}\right\|^{2}+\gamma_{n}\left\|u_{n}-x^{*}\right\|^{2} \\
& \leq \alpha_{n}\left\|f\left(W_{n} x_{n}\right)-x^{*}\right\|^{2}+\left\|x_{n}-x^{*}\right\|^{2}-\gamma_{n} r_{n}\left(2 \alpha-r_{n}\right)\left\|A y_{n}-A x^{*}\right\|^{2} .
\end{aligned}
$$

Hence, one has

$$
\begin{aligned}
& \gamma_{n} r_{n}\left(2 \alpha-r_{n}\right)\left\|A y_{n}-A x^{*}\right\|^{2} \\
& \leq \alpha_{n}\left\|f\left(W_{n} x_{n}\right)-x^{*}\right\|^{2}+\left\|x_{n}-x^{*}\right\|^{2}-\left\|x_{n+1}-x^{*}\right\|^{2} \\
& \leq \alpha_{n}\left\|f\left(W_{n} x_{n}\right)-x^{*}\right\|^{2}+\left\|x_{n}-x_{n+1}\right\|\left(\left\|x_{n}-x^{*}\right\|+\left\|x_{n+1}-x^{*}\right\|\right) .
\end{aligned}
$$

In view of the restrictions on $\left\{\alpha_{n}\right\}$ and $\left\{\gamma_{n}\right\}$, one finds that

$$
\lim _{n \rightarrow \infty}\left\|A y_{n}-A x^{*}\right\|=0 .
$$

On the other hand, one also has

$$
\begin{aligned}
& \left\|u_{n}-x^{*}\right\|^{2} \\
& \leq\left\langle\left(I-r_{n} A\right) y_{n}-\left(I-r_{n} A\right) x^{*}, u_{n}-x^{*}\right\rangle \\
& =\frac{1}{2}\left(\left\|\left(I-r_{n} A\right) y_{n}-\left(I-r_{n} A\right) x^{*}\right\|^{2}-\left\|y_{n}-u_{n}-r_{n}\left(A y_{n}-A x^{*}\right)\right\|^{2}+\left\|u_{n}-x^{*}\right\|^{2}\right) \\
& \leq \frac{1}{2}\left(\left\|y_{n}-x^{*}\right\|^{2}-\left\|y_{n}-u_{n}\right\|^{2}+2 r_{n}\left\|y_{n}-u_{n}\right\|\left\|A y_{n}-A x^{*}\right\|+\left\|u_{n}-x^{*}\right\|^{2}\right),
\end{aligned}
$$

which yields that

$$
\left\|u_{n}-x^{*}\right\|^{2} \leq\left\|x_{n}-x^{*}\right\|^{2}-\left\|y_{n}-u_{n}\right\|^{2}+2 r_{n}\left\|y_{n}-u_{n}\right\|\left\|A y_{n}-A x^{*}\right\| .
$$


It follows that

$$
\begin{aligned}
\left\|x_{n+1}-x^{*}\right\|^{2} \leq & \alpha_{n}\left\|f\left(W_{n} x_{n}\right)-x^{*}\right\|^{2}+\beta_{n}\left\|x_{n}-x^{*}\right\|^{2}+\gamma_{n}\left\|W_{n} \xi_{n}-x^{*}\right\|^{2} \\
\leq & \alpha_{n}\left\|f\left(W_{n} x_{n}\right)-x^{*}\right\|^{2}+\beta_{n}\left\|x_{n}-x^{*}\right\|^{2}+\gamma_{n}\left\|u_{n}-x^{*}\right\|^{2} \\
\leq & \alpha_{n}\left\|f\left(W_{n} x_{n}\right)-x^{*}\right\|^{2}+\left\|x_{n}-x^{*}\right\|^{2}-\gamma_{n}\left\|y_{n}-u_{n}\right\|^{2} \\
& +2 r_{n} \gamma_{n}\left\|y_{n}-u_{n}\right\|\left\|A y_{n}-A x^{*}\right\| .
\end{aligned}
$$

Hence,

$$
\begin{aligned}
\gamma_{n}\left\|y_{n}-u_{n}\right\|^{2} \leq & \alpha_{n}\left\|f\left(W_{n} x_{n}\right)-x^{*}\right\|^{2}+\left\|x_{n+1}-x_{n}\right\|\left(\left\|x_{n}-x^{*}\right\|+\left\|x_{n+1}-x^{*}\right\|\right) \\
& +2 r_{n} \gamma_{n}\left\|y_{n}-u_{n}\right\|\left\|A y_{n}-A x^{*}\right\| .
\end{aligned}
$$

This implies from (2.11) that $\lim _{n \rightarrow \infty}\left\|y_{n}-u_{n}\right\|=0$. In a similar way, one finds that $\lim _{n \rightarrow \infty}\left\|\xi_{n}-u_{n}\right\|=0$. Using (2.8), one has $\lim _{n \rightarrow \infty}\left\|W_{n} \xi_{n}-x_{n}\right\|=0$. This implies that $\lim _{n \rightarrow \infty}\left\|W_{n} \xi_{n}-\xi_{n}\right\|=0$. Notice that

$$
\frac{y_{n}-u_{n}}{r_{n}}-A y_{n} \in M u_{n}
$$

Let $\mu$ and $v$ be in $H$ such that $\mu \in M v$. Taking into account that $M$ is monotone, we find that

$$
\left\langle\frac{y_{n}-u_{n}}{r_{n}}-A y_{n}-\mu, u_{n}-v\right\rangle \geq 0 .
$$

This implies that

$$
\langle-A q-\mu, q-v\rangle \geq 0 .
$$

This implies that $-A q \in M q$, that is, $q \in(A+M)^{-1}(0)$.

Next, we prove that $q \in \cap_{i=1}^{\infty} F\left(S_{i}\right)$. We assume $q \notin \cap_{i=1}^{\infty} F\left(S_{i}\right)$, i.e., $W q \neq q$. Taking into account that $\xi_{n_{i}} \rightarrow q$ and the space satisfies Opial's condition, one has

$$
\begin{aligned}
\liminf _{i \rightarrow \infty}\left\|\xi_{n_{i}}-q\right\| & <\liminf _{i \rightarrow \infty}\left\|\xi_{n_{i}}-W q\right\| \\
& \leq \liminf _{i \rightarrow \infty}\left\{\left\|\xi_{n_{i}}-W \xi_{n_{i}}\right\|+\left\|W \xi_{n_{i}}-W q\right\|\right\} \\
& \leq \liminf _{i \rightarrow \infty}\left\{\left\|\xi_{n_{i}}-W \xi_{n_{i}}\right\|+\left\|\xi_{n_{i}}-q\right\|\right\} .
\end{aligned}
$$

From Lemma 1.2, we see that $\lim _{n \rightarrow \infty}\left\|W \xi_{n}-\xi_{n}\right\|=0$. This derives a contradiction. Thus, we have $q \in \cap_{i=1}^{\infty} F\left(S_{i}\right)$.

Now, we are in a position to show that $q \in E P(F)$. From $y_{n}=\operatorname{Res}_{s_{n}} x_{n}$, we see that

$$
\left\langle y-y_{n}, y_{n}-x_{n}\right\rangle \geq s_{n} F\left(y, u_{n}\right), \quad \forall y \in C .
$$

So, $0 \geq F(y, q)$. For $0<t \leq 1$ and $y \in C$, we set

$$
y_{t}=(1-t) q+t y .
$$

It follows that that $y_{t} \in C$. Hence, $0 \geq F\left(y_{t}, q\right)$. Taking into account the fact that

$$
t F\left(y_{t}, y\right) \geq t F\left(y_{t}, y\right)+(1-t) F\left(y_{t}, q\right) \geq F\left(y_{t}, y_{t}\right)=0,
$$

we arrive at $F(q, y) \geq 0, \forall y \in C$. This shows that $q \in E P(F)$. So,

$$
\limsup _{n \rightarrow \infty}\left\langle f(\bar{x})-\bar{x}, x_{n}-\bar{x}\right\rangle \leq 0 .
$$


Finally, we show that $x_{n} \rightarrow \bar{x}$ as $n \rightarrow \infty$. Note that

$$
\begin{aligned}
\left\|x_{n+1}-\bar{x}\right\|^{2} \leq & \beta_{n}\left\|x_{n}-\bar{x}\right\|\left\|x_{n+1}-\bar{x}\right\|+\gamma_{n}\left\|W_{n} \xi_{n}-\bar{x}\right\|\left\|x_{n+1}-\bar{x}\right\| \\
& +\alpha_{n}\left\langle f(\bar{x})-\bar{x}, x_{n+1}-\bar{x}\right\rangle+\alpha_{n}\left\langle f\left(W_{n} x_{n}\right)-f(\bar{x}), x_{n+1}-\bar{x}\right\rangle \\
\leq & \frac{1-\alpha_{n}(1-\kappa)}{2}\left\|x_{n}-\bar{x}\right\|^{2}+\frac{1}{2}\left\|x_{n+1}-\bar{x}\right\|^{2}+\alpha_{n}\left\langle f(\bar{x})-\bar{x}, x_{n+1}-\bar{x}\right\rangle,
\end{aligned}
$$

which implies that

$$
\left\|x_{n+1}-\bar{x}\right\|^{2} \leq\left(1-\alpha_{n}(1-\kappa)\right)\left\|x_{n}-\bar{x}\right\|^{2}+2 \alpha_{n}\left\langle f(\bar{x})-\bar{x}, x_{n+1}-\bar{x}\right\rangle .
$$

By virtue of Lemma 1.7, one obtains that $\lim _{n \rightarrow \infty}\left\|x_{n}-\bar{x}\right\|=0$. This completes the proof.

If $S_{i}$ is an identity mapping for each $i \geq 1$, we immediately find the following result.

Corollary 2.2. Let $H$ be a real Hilbert space and let $C$ be a nonempty convex closed subset of $H$. Let $f: C \rightarrow C$ be a $\kappa$-contraction. Let $A: C \rightarrow H$ be an $\alpha$-inverse-strongly monotone mapping and let $M: H \rightrightarrows H$ be a maximal monotone operator such that Dom $(M) \subset C$. Let $F$ be a bifunction from $C \times C$ to $\mathbb{R}$ which satisfies (A1)-(A4). Let $\left\{x_{n}\right\}$ be a sequence generated in the process: $x_{1} \in C$ and

$$
\left\{\begin{array}{l}
u_{n}=J_{r_{n}}\left(y_{n}-r_{n} A y_{n}\right), \\
x_{n+1}=\alpha_{n} f\left(x_{n}\right)+\beta_{n} x_{n}+\gamma_{n} J_{r_{n}}\left(u_{n}-r_{n} A u_{n}\right), \quad \forall n \geq 1,
\end{array}\right.
$$

where $y_{n}$ is in $C$ such that $s_{n} F\left(y_{n}, y\right)+\left\langle y-y_{n}, y_{n}-x_{n}\right\rangle \geq 0, \forall y \in C,\left\{\alpha_{n}\right\},\left\{\beta_{n}\right\}$ and $\left\{\gamma_{n}\right\}$ are sequences in $(0,1)$ such that $\alpha_{n}+\beta_{n}+\gamma_{n}=1$ and $\left\{r_{n}\right\}$ and $\left\{s_{n}\right\}$ are positive number sequences. Assume that the above control sequences satisfy the following restrictions: $\lim _{n \rightarrow \infty}\left|s_{n}-s_{n+1}\right|=\lim _{n \rightarrow \infty}\left|r_{n}-r_{n+1}\right|=0$, $0<\liminf _{n \rightarrow \infty} \beta_{n} \leq \limsup _{n \rightarrow \infty} \beta_{n}<1, \lim _{n \rightarrow \infty} \alpha_{n}=0$ and $\sum_{n=1}^{\infty} \alpha_{n}=\infty, 0<r \leq r_{n} \leq r^{\prime}<2 \alpha, 0<s \leq$ $s_{n} \leq s^{\prime}<2 \beta$, where $r, r^{\prime}$, s and $s^{\prime}$ are four real numbers. If $(A+M)^{-1}(0) \cap E P(F) \neq \emptyset$. Then $\left\{x_{n}\right\}$ converges strongly to $\bar{x}=\operatorname{Proj}_{(A+M)^{-1}(0) \cap E P(F)} f(\bar{x})$.

Let $I_{C}$ be the indicator function of $C$, i.e.,

$$
I_{C}(x)= \begin{cases}0, & x \in C, \\ +\infty, & x \notin C .\end{cases}
$$

Since $I_{C}$ is a proper lower semicontinuous convex function on $H$, we see that the subdifferential $\partial I_{C}$ of $I_{C}$ is a maximal monotone operator. It is clearly that $J_{r} x=\operatorname{Proj}_{C} x, \forall x \in H$. From Corollary 2.2, the following result is not hard to derive.

Corollary 2.3. Let $H$ be a real Hilbert space and let $C$ be a nonempty convex closed subset of $H$. Let $f: C \rightarrow C$ be a $\kappa$-contraction. Let $A: C \rightarrow H$ be an $\alpha$-inverse-strongly monotone mapping and let $F$ be a bifunction from $C \times C$ to $\mathbb{R}$ which satisfies (A1)-(A4). Let $\left\{x_{n}\right\}$ be a sequence generated in the process: $x_{1} \in C$ and

$$
\left\{\begin{array}{l}
u_{n}=\operatorname{Proj}_{C}\left(y_{n}-r_{n} A y_{n}\right), \\
x_{n+1}=\alpha_{n} f\left(x_{n}\right)+\beta_{n} x_{n}+\gamma_{n} \operatorname{Proj}_{C}\left(u_{n}-r_{n} A u_{n}\right), \quad \forall n \geq 1,
\end{array}\right.
$$

where $y_{n}$ is in $C$ such that $s_{n} F\left(y_{n}, y\right)+\left\langle y-y_{n}, y_{n}-x_{n}\right\rangle \geq 0, \forall y \in C,\left\{\alpha_{n}\right\},\left\{\beta_{n}\right\}$ and $\left\{\gamma_{n}\right\}$ are sequences in $(0,1)$ such that $\alpha_{n}+\beta_{n}+\gamma_{n}=1$ and $\left\{r_{n}\right\}$ and $\left\{s_{n}\right\}$ are positive number sequences. Assume that the above control sequences satisfy the following restrictions: $\lim _{n \rightarrow \infty}\left|s_{n}-s_{n+1}\right|=\lim _{n \rightarrow \infty}\left|r_{n}-r_{n+1}\right|=0$, 
$0<\liminf _{n \rightarrow \infty} \beta_{n} \leq \limsup _{n \rightarrow \infty} \beta_{n}<1, \lim _{n \rightarrow \infty} \alpha_{n}=0$ and $\sum_{n=1}^{\infty} \alpha_{n}=\infty, 0<r \leq r_{n} \leq r^{\prime}<2 \alpha, 0<s \leq$ $s_{n} \leq s^{\prime}<2 \beta$, where $r, r^{\prime}, s$ and $s^{\prime}$ are four real numbers. If $V I(C, A) \cap E P(F) \neq \emptyset$. Then $\left\{x_{n}\right\}$ converges strongly to $\bar{x}=\operatorname{Proj}_{V I(C, A) \cap E P(F)} f(\bar{x})$.

\section{Acknowledgements}

The author thanks the two referees for their valuable comments and suggestions. This paper was partially supported by the Natural Science Foundation of Zhejiang Province under Grant No. LY16F020014.

\section{REFERENCES}

[1] D.H. Peaceman, H.H. Rachford, The numerical solution of parabolic and elliptic differential equations, J. Soc. Industrial Appl. Math. 3 (1995), 28-41.

[2] J. Douglas, H.H. Rachford, On the numerical solution of heat conduction problems in two and three space variables, Trans. Amer. Math. Soc. 82 (1956), 421-439.

[3] X. Qin, S.Y. Cho, Convergence analysis of a monotone projection algorithm in reflexive Banach spaces, Acta Math. Sci. 37 (2017), 488-502.

[4] S. Wang, W. Zhu, Sparse graph embedding unsupervised feature selection, IEEE Trans. Sys. Man Cybernet. 48 (2018), 329-341.

[5] Z. Cai, W. Zhu, Multi-label feature selection via feature manifold learning and sparsity regularization, Int. J. Mach. Learn. Cybernet. 9 (2018), 1321-1334.

[6] N.T.T. Van, J.J. Strodiot, V.H. Nguyen, P.T. Vuong, An extragradient-type method for solving nonmonotone quasiequilibrium problems, Optimization, 67 (2018), 651-664.

[7] B.A. Bin Dehaish, et al., Weak and strong convergence of algorithms for the sum of two accretive operators with applications, J. Nonlinear Convex Anal. 16 (2015), 1321-1336.

[8] X. Qin, J.C. Yao, Projection splitting algorithms for nonself operators, J. Nonlinear Convex Anal. 18 (2017), $925-935$.

[9] S.Y. Cho, Generalized mixed equilibrium and fixed point problems in a Banach space, J. Nonlinear Sci. Appl. 9 (2016), 1083-1092.

[10] S.Y. Cho, X. Qin, On the strong convergence of an iterative process for asymptotically strict pseudocontractions and equilibrium problems, Appl. Math. Comput. 235 (2014), 430-438.

[11] S.S. Chang, H.W.J. Lee, C.K. Chan, A new method for solving equilibrium problem fixed point problem and variational inequality problem with application to optimization, Nonlinear Anal. 70 (2009), 3307-3319.

[12] S.S. Chang, L. Wang, Y. Zhao, On a class of split equality fixed point problems in Hilbert spaces, J. Nonlinear Var. Anal. 1 (2017), 201-212.

[13] S.Y. Cho, B.A. Bin Dehaish, X. Qin, Weak convergence of a splitting algorithm in Hilbert spaces, J. Appl. Anal. Comput. 7 (2017), 427-438.

[14] S.Y. Cho, X. Qin, L. Wang, Strong convergence of a splitting algorithm for treating monotone operators, Fixed Point Theory Appl. 2014 (2014), Article ID 94.

[15] A. Gibali, Two simple relaxed perturbed extragradient methods for solving variational inequalities in Euclidean spaces, J. Nonlinear Var. Anal. 2 (2018), 49-61.

[16] X. Qin, J.C. Yao, Weak convergence of a Mann-like algorithm for nonexpansive and accretive operators, J. Inequal. Appl. 2016 (2016), Article ID 232.

[17] O.M. Romanus, U.V. Nnyaba, M.O. Nnakwe, Algorithms for a system of generalized mixed equilibrium problems and a countable family of some nonlinear multi-valued nonexpansive-type maps, Acta Math. Scientia 38 (2018), 1805-1820.

[18] L.D. Muu, X.T. Le, A splitting algorithm for finding fixed points of nonexpansive mappings and solving equilibrium problems, J. Fixed Point Theory Appl. 20 (2018), Article ID 130.

[19] C. Zhang, Y. Wang, Proximal algorithm for solving monotone variational inclusion, Optimization, 67 (2018), 1197-1209.

[20] K. Shimoji, W. Takahashi, Strong convergence to common fixed points of infinite nonexpansive mappings and applications, Taiwanese J. Math. 5 (2001) 387-404. 
[21] L. Liu, A common fixed point theorem in generalized Menger metric spaces, Commun. Optim. Theory 2018 (2018), Article ID 9.

[22] W. Takahashi, C.F. Wen, J.C. Yao, An implicit algorithm for the split common fixed point problem in Hilbert spaces and applications, Appl. Anal. Optim. 1 (2017), 423-439.

[23] X. Qin, S.Y. Cho, L. Wang, Iterative algorithms with errors for zero points of m-accretive operators, Fixed Point Theory Appl. 2014 (2014), Article ID 148.

[24] T. Suzuki, Strong convergence theorems for infinite families of nonexpansive mappings in general Banach spaces, Fixed Point Theory Appl. 2005 (2005), Article ID 685918.

[25] R.B. Kellogg, Nonlinear alternating direction algorithm, Math. Comput. 23 (1969), 23-28.

[26] P.L. Lions, B. Mercier, Splitting algorithms for the sum of two nonlinear operators, SIAM J. Numer. Anal. 16 (1979), 964-979.

[27] E. Blum, W. Oettli, From optimization and variational inequalities to equilibrium problems, Math. Stud. 63 (1994), 123145.

[28] V. Barbu, Nonlinear Semigroups and Differential Equations in Banach Spaces, Noordhoff, Amsterdam, 1976.

[29] X. Qin, S.Y. Cho, L. Wang, Strong convergence of an iterative algorithm involving nonlinear mappings of nonexpansive and accretive type, Optimization, 67 (2018), 1377-1388.

[30] Z. Xue, H. Zhou, Y.J. Cho, Iterative solutions of nonlinear equations for m-accretive operators in Banach spaces, J. Nonlinear Convex Anal. 1 (2000), 313-320. 